Title: $\quad$ Derivative based global sensitivity measures

Name: $\quad$ Sergeï Kucherenko ${ }^{1}$ and Bertrand Iooss ${ }^{2,3}$

Affil./Addr. 1: Imperial College London

London, SW7 2AZ, UK

E-mail: s.kucherenko@imperial.ac.uk

Affil./Addr. 2: $\quad$ EDF R\&D

6 quai Watier, 78401 Chatou, France

E-mail: bertrand.iooss@edf.fr

Affil./Addr. 3: $\quad$ Institut de Mathématiques de Toulouse

Université Paul Sabatier

118 route de Narbonne, 31062 Toulouse, France

\title{
Derivative based global sensitivity
}

\section{measures}

\section{Abstract}

The method of derivative based global sensitivity measures (DGSM) has recently become popular among practitioners. It has a strong link with the Morris screening method and Sobol' sensitivity indices and has several advantages over them. DGSM are very easy to implement and evaluate numerically. The computational time required for numerical evaluation of DGSM is generally much lower than that for estimation of Sobol' sensitivity indices. This paper presents a survey of recent advances in DGSM concerning lower and upper bounds on the values of Sobol' total sensitivity indices $S_{i}^{\text {tot }}$. Using these bounds it is possible in most cases to get a good practical estimation of the values of $S_{i}^{\text {tot }}$. Several examples are used to illustrate an application of DGSM. 
Keywords: Sensitivity analysis, Sobol' indices, Morris method, Model derivatives, DGSM, Poincaré inequality

\section{Introduction}

Global sensitivity analysis (SA) offers a comprehensive approach to the model analysis. Unlike local SA, global SA methods evaluate the effect of a factor while all other factors are varied as well and thus they account for interactions between variables and do not depend on the choice of a nominal point. Reviews of different global SA methods can be found in Saltelli et al [30] and Sobol and Kucherenko [37]. The method of global sensitivity indices suggested by Sobol [33, 34], and then further developed by Homma and Saltelli [11] is one of the most efficient and popular global SA techniques. It belongs to the class of variance-based methods. These methods provide information on the importance of different subsets of input variables to the output variance. There are two types of Sobol' sensitivity indices: the main effect indices, which estimate the individual contribution of each input parameter to the output variance, and the total sensitivity indices, which measure the total contribution of a single input factor or a group of inputs. The total sensitivity indices are used to identify non-important variables which can then be fixed at their nominal values to reduce model complexity. This approach is known as "factors' fixing setting" [30]. For high-dimensional models the direct application of variance-based global SA measures can be extremely timeconsuming and impractical.

A number of alternative SA techniques have been proposed. One of them is the screening method by Morris [21]. It can be regarded as global as the final measure is obtained by averaging local measures (the elementary effects). This method is considerably cheaper than the variance based methods in terms of computational time. The Morris method can be used for identifying unimportant variables. However, the Morris 
method has two main drawbacks. Firstly, it uses random sampling of points from the fixed grid (levels) for averaging elementary effects which are calculated as finite differences with the increment delta comparable with the range of uncertainty. For this reason it can not correctly account for the effects with characteristic dimensions much less than delta. Secondly, it lacks the ability of the Sobol' method to provide information about main effects (contribution of individual variables to uncertainty) and it can't distinguish between low and high order interactions.

This paper presents a survey of derivative based global sensitivity measures (DGSM) and their link with Sobol' sensitivity indices. DGSM are based on averaging local derivatives using Monte Carlo or Quasi Monte Carlo sampling methods. This technique is much more accurate than the Morris method as the elementary effects are evaluated as strict local derivatives with small increments compared to the variable uncertainty ranges. Local derivatives are evaluated at randomly or quasi randomly selected points in the whole range of uncertainty and not at the points from a fixed grid.

The so-called alternative global sensitivity estimator defined as a normalized integral of partial derivatives was firstly introduced by Sobol and Gershman 36]. Kucherenko et al [17] introduced some other DGSM and coined the acronym DGSM. They showed that DGSM can be seen as the generalization of the Morris method [21]. Kucherenko et al [17] also established empirically the link between DGSM and Sobol' sensitivity indices. They showed that the computational cost of numerical evaluation of DGSM can be much lower than that for estimation of Sobol' sensitivity indices.

Sobol and Kucherenko 38 , proved theoretically that, in the cases of uniformly and normally distributed input variables, there is a link between DGSM and the Sobol' total sensitivity index $S_{i}^{\text {tot }}$ for the same input. They showed that DGSM can be used as an upper bound on total sensitivity index $S_{i}^{\text {tot }}$. Small values of DGSM imply small 
$S_{i}^{\text {tot }}$, and hence unessential factors $x_{i}$. However, ranking influential factors using DGSM can be similar to that based on $S_{i}^{\text {tot }}$ only for the case of linear and quasi-linear models. For highly non-linear models two rankings can be very different. They also introduced modified DGSM which can be used for both a single input and groups of inputs [39]. From DGSM, Kucherenko and Song [16] have also derived lower bounds on total sensitivity index. Lamboni et al [19] extended results of Sobol' and Kucherenko for models with input variables belonging to the general class of continuous probability distributions. In the same framework, Roustant et al [28] have defined crossed-DGSM, based on second-order derivatives of model output, in order to bound the total Sobol' indices of an interaction between two inputs.

All these DGSM measures can be applied for problems with a high number of input variables to reduce the computational time. Indeed, the numerical efficiency of the DGSM method can be improved by using the automatic differentiation algorithm for calculation DGSM as was shown in Kiparissides et al [15]. However, the number of required function evaluations still remains to be proportional to the number of inputs. This dependence can be greatly reduced using an approach based on algorithmic differentiation in the adjoint or reverse mode [9] (Variational Methods). It allows estimating all derivatives at a cost at most 4-6 times of that for evaluating the original function [13].

This paper is organised as follows: the Morris method and DGSM are firstly described in the following section. Sobol' global sensitivity indices and useful relationships are then introduced. Therefore, DGSM-based lower and uppers bounds on total Sobol' sensitivity indices for uniformly and normally distributed random variables are presented, followed by DGSM for groups of variables and their link with total Sobol' sensitivity indices. Another section presents the upper bounds results in the general case of variables with continuous probability distributions. Then, computational costs 
are considered, followed by some test cases which illustrate an application of DGSM and their links with total Sobol' sensitivity indices. Finally, conclusions are presented in the last section.

\section{From Morris method to DGSM}

\section{Basics of the Morris method}

The Morris method is traditionally used as a screening method for problems with a high number of variables for which function evaluations can be CPU-time consuming (see Design of Experiments for Screening). It is composed of individually randomized 'one-factor-at-a-time' (OAT) experiments. Each input factor may assume a discrete number of values, called levels, which are chosen within the factor range of variation.

The sensitivity measures proposed in the original work of Morris [21] are based on what is called an elementary effect. It is defined as follows. The range of each input variable is divided into $p$ levels. Then the elementary effect (incremental ratio) of the $i$-th input factor is defined as

$$
E E_{i}\left(\mathbf{x}^{*}\right)=\frac{\left[G\left(x_{1}^{*}, \ldots, x_{i-1}^{*}, x_{i}^{*}+\Delta, x_{i+1}^{*}, \ldots, x_{d}^{*}\right)-G\left(\mathbf{x}^{*}\right)\right]}{\Delta}
$$

where $\Delta$ is a predetermined multiple of $1 /(p-1)$ and point $\mathbf{x}^{*}=\left(x_{1}^{*}, \ldots, x_{d}^{*}\right) \in H^{d}$ is such that $x_{i}^{*}+\Delta \leq 1$. One can see that the elementary effect are finite difference approximations of the model derivative with respect to $x_{i}$ and using a large perturbation step $\Delta$.

The distribution of elementary effects $E E_{i}$ is obtained by randomly sampling $R$ points from $H^{d}$. Two sensitivity measures are evaluated for each factor: $\mu_{i}$ an estimate of the mean of the distribution $E E_{i}$, and $\sigma_{i}$ an estimate of the standard deviation of $E E_{i}$. A high value of $\mu_{i}$ indicates an input variable with an important overall influence 
on the output. A high value of $\sigma_{i}$ indicates a factor involved in interaction with other factors or whose effect is nonlinear. The computational cost of the Morris method is $N_{F}=R(d+1)$.

The revised version of the $E E_{i}\left(\mathbf{x}^{*}\right)$ measure and a more effective sampling strategy, which allows a better exploration of the space of the uncertain input factors was proposed by Campolongo et al [3]. To avoid the canceling effect which appears in non-monotonic functions Campolongo et al [3] introduced another sensitivity measure $\mu_{i}^{*}$ based on the absolute value of $E E_{i}\left(\mathbf{x}^{*}\right):\left|E E_{i}\left(\mathbf{x}^{*}\right)\right|$. It was also noticed that $\mu_{i}^{*}$ has similarities with the total sensitivity index $S_{i}^{\text {tot }}$ in that it can give a ranking of the variables similar to that based on the $S_{i}^{\text {tot }}$ but no formal proof of the link between $\mu_{i}^{*}$ and $S_{i}^{\text {tot }}$ was given $[3$.

Finally, other extensions of the initial Morris method have been introduced for the second-order effects' analysis [2] [4] [6], for the estimation of Morris' measures with any-type of design [26] [32] and for building some 3D Morris' graph [26].

\section{The local sensitivity measure}

Consider a differentiable function $G(\mathbf{x})$, where $\mathbf{x}=\left(x_{1}, \ldots, x_{d}\right)$ is a vector of input variables defined in the unit hypercube $H^{d}\left(0 \leq x_{i} \leq 1, i=1, \ldots, d\right)$. Local sensitivity measures are based on partial derivatives

$$
E_{i}\left(\mathbf{x}^{*}\right)=\frac{\partial G\left(\mathbf{x}^{*}\right)}{\partial x_{i}}
$$

This measure $E_{i}$ is the limit version of the elementary effect $E E_{i}$ defined in (2) when $\Delta$ tends to zero. It is its generalization in this sense. In SA, using the partial derivative $\partial G / \partial x_{i}$ is well known as a local method (see Variational Methods). In this paper, the goal is to take advantage of this information in global SA.

The local sensitivity measure $E_{i}\left(\mathbf{x}^{*}\right)$ depends on a nominal point $\mathbf{x}^{*}$ and it changes with a change of $\mathbf{x}^{*}$. This deficiency can be overcome by averaging $E_{i}\left(\mathbf{x}^{*}\right)$ over 
the parameter space $H^{d}$. This is done just below, allowing to define new sensitivity measures, called DGSM for Derivative-based Global Sensitivity Measures.

\section{DGSM for uniformly distributed variables}

Assume that $\partial G / \partial x_{i} \in L_{2}$. Three different DGSM measures are defined:

$$
\begin{gathered}
\nu_{i}=\int_{H^{d}}\left(\frac{\partial G(\mathbf{x})}{\partial x_{i}}\right)^{2} d \mathbf{x}, \\
w_{i}^{(m)}=\int_{H^{d}} x_{i}^{m} \frac{\partial G(\mathbf{x})}{\partial x_{i}} d \mathbf{x},
\end{gathered}
$$

where $m>0$ is a constant, and

$$
\varsigma_{i}=\frac{1}{2} \int_{H^{d}} x_{i}\left(1-x_{i}\right)\left(\frac{\partial G(\mathbf{x})}{\partial x_{i}}\right)^{2} d \mathbf{x} .
$$

\section{DGSM for randomly distributed variables}

Consider a function $G\left(X_{1}, \ldots, X_{d}\right)$, where $X_{1}, \ldots, X_{d}$ are independent random variables, defined in the Euclidian space $R^{d}$, with cumulative density functions (cdfs) $F_{1}\left(x_{1}\right), \ldots, F_{d}\left(x_{d}\right)$. The following DGSM was introduced in Sobol and Kucherenko [38]:

$$
\nu_{i}=\int_{R^{d}}\left(\frac{\partial G(\mathbf{x})}{\partial x_{i}}\right)^{2} d F(\mathbf{x})=\mathbb{E}\left[\left(\frac{\partial G(\mathbf{x})}{\partial x_{i}}\right)^{2}\right],
$$

with $F$ the joint cdf. A new measure is also introduced:

$$
w_{i}=\int_{R^{d}} \frac{\partial G(\mathbf{x})}{\partial x_{i}} d F(\mathbf{x})=\mathbb{E}\left(\frac{\partial G(\mathbf{x})}{\partial x_{i}}\right) .
$$

In (3) and (6), $\nu_{i}$ is in fact the mean value of $\left(\partial G / \partial x_{i}\right)^{2}$. In the following and in practice, it will be the most useful DGSM.

\section{Sobol' global sensitivity indices}

\section{Definitions}

The method of global sensitivity indices developed by Sobol' (see Variance-based Sensitivity Analysis: Theory and Estimation Algorithms) is based on ANOVA decom- 
position [10]. Consider a square integrable function $G(\mathbf{x})$ defined in the unit hypercube $H^{d}$. It can be expanded in the following form

$$
G(\mathbf{x})=g_{0}+\sum_{i} g_{i}\left(x_{i}\right)+\sum_{i<j} g_{i j}\left(x_{i}, x_{j}\right)+\ldots+g_{12 \ldots d}\left(x_{1}, x_{2}, \ldots, x_{d}\right) .
$$

This decomposition is unique if conditions $\int_{0}^{1} g_{i_{1} \ldots i_{s}} d x_{i_{k}}=0 \quad$ for $1 \leq k \leq s$, are satisfied. Here $1 \leq i_{1}<\cdots<i_{s} \leq d$.

The variances of the terms in the ANOVA decomposition add up to the total variance of the function

$$
V=\sum_{s=1}^{d} \sum_{i_{1}<\cdots<i_{s}}^{d} V_{i_{1} \ldots i_{s}}
$$

where $V_{i_{1} \ldots i_{s}}=\int_{0}^{1} g_{i_{1} \ldots i_{s}}^{2}\left(x_{i_{1}}, \ldots, x_{i_{s}}\right) d x_{i_{1}}, \ldots, x_{i_{s}}$ are called partial variances.

Sobol' defined the global sensitivity indices as the ratios

$$
S_{i_{1} \ldots i_{s}}=V_{i_{1} \ldots i_{s}} / V
$$

All $S_{i_{1} \ldots i_{s}}$ are non negative and add up to one:

$$
\sum_{i=1}^{d} S_{i}+\sum_{i} \sum_{j} S_{i j}+\sum_{i} \sum_{j} \sum_{k} S_{i j k} \ldots+S_{1,2, \ldots, d}=1 .
$$

Sobol' also defined sensitivity indices for subsets of variables. Consider two complementary subsets of variables $y$ and $z$ :

$$
\mathbf{x}=(y, z)
$$

Let $y=\left(x_{i_{1}}, \ldots, x_{i_{m}}\right), 1 \leq i_{1}<\ldots<i_{m} \leq d, K=\left(i_{1}, \ldots, i_{m}\right)$. The variance corresponding to the set $y$ is defined as

$$
V_{y}=\sum_{s=1}^{m} \sum_{\left(i_{1}<\cdots<i_{s}\right) \in K} V_{i_{1} \ldots i_{s}} .
$$

$V_{y}$ includes all partial variances $V_{i_{1}}, V_{i_{2}}, \ldots, V_{i_{1} \ldots i_{s}}$ such that their subsets of indices $\left(i_{1}, \ldots, i_{s}\right) \in K$ 
The total sensitivity indices were introduced by Homma and Saltelli [11]. The total variance $V_{y}^{\text {tot }}$ is defined as

$$
V_{y}^{\text {tot }}=V-V_{z}
$$

$V_{y}^{\text {tot }}$ consists of all $V_{i_{1} \ldots i_{s}}$ such that at least one index $i_{p} \in K$ while the remaining indices can belong to the complimentary to $K$ set $\bar{K}$. The corresponding global sensitivity indices are defined as

$$
\begin{aligned}
& S_{y}=V_{y} / V, \\
& S_{y}^{\text {tot }}=V_{y}^{\text {tot }} / V .
\end{aligned}
$$

The important indices in practice are $S_{i}$ and $S_{i}^{\text {tot }}, i=1, \ldots, d$ :

$$
\begin{aligned}
& S_{i}=V_{i} / V, \\
& S_{i}^{\mathrm{tot}}=V_{i}^{\mathrm{tot}} / V .
\end{aligned}
$$

Their values in most cases provide sufficient information to determine the sensitivity of the analyzed function to individual input variables. Variance-based methods generally require a large number of function evaluations (see Variance-based Methods: Theory and Algorithms) to achieve reasonable convergence and can become impractical for large engineering problems.

\section{Useful relationships}

To present further results on lower and upper bounds of $S_{i}^{\text {tot }}$, new notations and useful relationships have to be firstly presented. Denote $u_{i}(\mathbf{x})$ the sum of all terms in the ANOVA decomposition (8) that depend on $x_{i}$ :

$$
u_{i}(\mathbf{x})=g_{i}\left(x_{i}\right)+\sum_{j=1, j \neq i}^{d} g_{i j}\left(x_{i}, x_{j}\right)+\cdots+g_{12 \cdots d}\left(x_{1}, \cdots, x_{d}\right) .
$$

From the definition of ANOVA decomposition it follows that

$$
\int_{H^{d}} u_{i}(\mathbf{x}) d \mathbf{x}=0 .
$$


It is obvious that

$$
\frac{\partial G}{\partial x_{i}}=\frac{\partial u_{i}}{\partial x_{i}}
$$

Denote $\mathbf{z}=\left(x_{1}, \ldots, x_{i-1}, x_{i+1}, \ldots, x_{d}\right)$ the vector of all variables but $x_{i}$, then $\mathbf{x} \equiv\left(x_{i}, \mathbf{z}\right)$ and $G(\mathbf{x}) \equiv G\left(x_{i}, \mathbf{z}\right)$. The ANOVA decomposition of $G(\mathbf{x})$ (8) can be presented in the following form

$$
G(\mathbf{x})=u_{i}\left(x_{i}, \mathbf{z}\right)+v(\mathbf{z}),
$$

where $v(\mathbf{z})$ is the sum of terms independent of $x_{i}$. Because of 12 it is easy to show that $v(\mathbf{z})=\int_{0}^{1} G(\mathbf{x}) d x_{i}$. Hence

$$
u_{i}\left(x_{i}, \mathbf{z}\right)=G(\mathbf{x})-\int_{0}^{1} G(\mathbf{x}) d x_{i} .
$$

This equation can be found in Lamboni [18]. The total partial variance $V_{i}^{\text {tot }}$ can be computed as

$$
V_{i}^{\mathrm{tot}}=\int_{H^{d}} u_{i}^{2}(\mathbf{x}) d \mathbf{x}=\int_{H^{d}} u_{i}^{2}\left(x_{i}, z\right) d x_{i} d \mathbf{z} .
$$

Then the total sensitivity index $S_{i}^{\text {tot }} 10$ is equal to

$$
S_{i}^{\mathrm{tot}}=\frac{1}{V} \int_{H^{d}} u_{i}^{2}(\mathbf{x}) d \mathbf{x} .
$$

\section{A first direct link between total Sobol' sensitivity indices and partial derivatives}

Consider continuously differentiable function $G(\mathbf{x})$ defined in the unit hypercube $H^{d}=[0,1]^{d}$. This section presents a theorem that establishes links between the index $S_{i}^{\text {tot }}$ and the limiting values of $\left|\partial G / \partial x_{i}\right|$.

In the case when $\mathbf{y}=\left(x_{i}\right)$, Sobol'-Jansen formula [14] 35] 31] for $D_{i}^{\text {tot }}$ can be rewritten as

$$
D_{i}^{\text {tot }}=\frac{1}{2} \int_{H^{d}} \int_{0}^{1}[G(\mathbf{x})-G(\stackrel{\circ}{\mathbf{x}})]^{2} d \mathbf{x} d x_{i}^{\prime},
$$

where $\stackrel{o}{\mathbf{x}}=\left(x_{1}, \ldots, x_{i-1}, x_{i}^{\prime}, x_{i+1}, \ldots, x_{n}\right)$.

Theorem 1. Assume that $c \leq\left|\frac{\partial G}{\partial x_{i}}\right| \leq C$, then 


$$
\frac{c^{2}}{12 V} \leq S_{i}^{\text {tot }} \leq \frac{C^{2}}{12 V}
$$

Proof: Consider the increment of $G(\mathbf{x})$ in $(16)$ :

$$
G(\mathbf{x})-G(\stackrel{\circ}{\mathbf{x}})=\frac{\partial G(\hat{\mathbf{x}})}{\partial x_{i}}\left(x_{i}-x_{i}^{\prime}\right)
$$

where $\hat{\mathbf{x}}$ is a point between $\mathbf{x}$ and $\stackrel{\circ}{\mathbf{x}}$. Substituting $(18)$ into $(16)$ leads to

$$
V_{i}^{\mathrm{tot}}=\frac{1}{2} \int_{H^{d}} \int_{0}^{1}\left(\frac{\partial G(\hat{\mathbf{x}})}{\partial x_{i}}\right)^{2}\left(x_{i}-x_{i}^{\prime}\right)^{2} d \mathbf{x} d x_{i}^{\prime} .
$$

In (19) $c^{2} \leq\left(\partial G / \partial x_{i}\right)^{2} \leq C^{2}$ while the remaining integral is

$$
\int_{0}^{1} \int_{0}^{1}\left(x_{i}^{\prime}-x_{i}\right)^{2} d x_{i}^{\prime} d x_{i}=\frac{1}{6}
$$

Thus obtained inequalities are equivalent to 17$)$. Consider the function $G=g_{0}+c\left(x_{i}-\right.$ $1 / 2$ ). In this case $C=c, V=1 / 12$ and $S_{i}^{\text {tot }}=1$ and the inequalities in (17) become equalities.

\section{DGSM-based bounds for uniformly and normally distributed variables}

In this section, several theorems are listed in order to define useful lower and upper bounds of the total Sobol' indices. The proofs of these theorems come from previous works and papers and are not recalled here. Two cases are considered: variables $\mathbf{x}$ following uniform distributions and variables $\mathbf{x}$ following Gaussian distributions. The general case will be seen in a subsequent section.

\section{Uniformly distributed variables}

\section{Lower bounds on $S_{i}^{\text {tot }}$}

Theorem 2. There exists the following lower bound between DGSM (3) and the Sobol' total sensitivity index: 


$$
\frac{\left(\int_{H^{d}}[G(1, \mathbf{z})-G(0, \mathbf{z})][G(1, \mathbf{z})+G(0, \mathbf{z})-2 G(\mathbf{x})] d \mathbf{x}\right)^{2}}{4 \nu_{i} V}<S_{i}^{\text {tot }}
$$

Proof: The proof of this Theorem is given in Kucherenko and Song [16] and is based on equation (15) and a Cauchy-Schwartz inequality applied on $\int_{H^{d}} u_{i}(\mathbf{x}) \frac{\partial u_{i}(\mathbf{x})}{\partial x_{i}} d \mathbf{x}$.

The lower bound number number one (LB1) is defined as

$$
\frac{\left(\int_{H^{d}}[G(1, \mathbf{z})-G(0, \mathbf{z})][G(1, \mathbf{z})+G(0, \mathbf{z})-2 G(\mathbf{x})] d \mathbf{x}\right)^{2}}{4 \nu_{i} V} .
$$

Theorem 3. There exists the following lower bound, denoted $\gamma(m)$, between DGSM (4) and the Sobol' total sensitivity index:

$$
\gamma(m)=\frac{(2 m+1)\left[\int_{H^{d}}(G(1, \mathbf{z})-G(\mathbf{x})) d \mathbf{x}-w_{i}^{(m+1)}\right]^{2}}{(m+1)^{2} V}<S_{i}^{\text {tot }} .
$$

Proof: The proof of this Theorem in given in Kucherenko and Song [16] and is based on equation (15) and a Cauchy-Schwartz inequality applied on $\int_{H^{d}} x_{i}^{m} u_{i}(\mathbf{x}) d \mathbf{x}$.

In fact, Theorem 3 gives a set of lower bounds depending on parameter $m$. The value of $m$ at which $\gamma(m)$ attains its maximum is of particular interest. Further, star $\left(^{*}\right)$ is used to denote such a value $m: m^{*}=\arg \max (\gamma(m)) \cdot \gamma\left(m^{*}\right)$ is called the lower bound number two (LB2):

$$
\gamma\left(m^{*}\right)=\frac{\left(2 m^{*}+1\right)\left[\int_{H^{d}}(G(1, \mathbf{z})-G(\mathbf{x})) d \mathbf{x}-w_{i}^{\left(m^{*}+1\right)}\right]^{2}}{\left(m^{*}+1\right)^{2} V}
$$

The maximum lower bound $\mathrm{LB}^{*}$ is defined as

$$
\mathrm{LB}^{*}=\max (\mathrm{LB} 1, \mathrm{LB} 2)
$$

Both lower and upper bounds can be estimated by a set of derivative based measures:

$$
\Upsilon_{i}=\left\{\nu_{i}, w_{i}^{(m)}, \zeta_{i}\right\}, m>0
$$




\section{Upper bounds on $S_{i}^{\text {tot }}$}

Theorem 4. There exists the following upper bound between DGSM (3) and the Sobol' total sensitivity index:

$$
S_{i}^{\text {tot }} \leq \frac{\nu_{i}}{\pi^{2} V}
$$

Proof: The proof of this Theorem in given in Sobol and Kucherenko [38]. It is based on inequality:

$$
\int_{0}^{1} u^{2}(x) d x \leq \frac{1}{\pi^{2}} \int_{0}^{1}\left(\frac{\partial u}{\partial x}\right)^{2} d x
$$

and relationships 13 and 15 .

Consider the set of values $\nu_{1}, \ldots, \nu_{d}, 1 \leq i \leq d$. One can expect that smaller $\nu_{i}$ correspond to less influential variables $x_{i}$. This importance criterion is similar to the modified Morris importance measure $\mu^{*}$, whose limiting values are

$$
\mu_{i}^{*}=\int_{H^{d}}\left|\frac{\partial G(\mathbf{x})}{\partial x_{i}}\right| d \mathbf{x}
$$

From a practical point of view the criteria $\mu_{i}$ and $\nu_{i}$ are equivalent: they are evaluated by the same numerical algorithm and are linked by relations $\nu_{i} \leq C \mu_{i}$ and $\mu_{i} \leq \sqrt{\nu_{i}}$

The right term in (25) is further called the upper bound number one (UB1).

Theorem 5. There exists the following upper bound between DGSM (5) and the Sobol' total sensitivity index:

$$
S_{i}^{\text {tot }} \leq \frac{\varsigma_{i}}{V}
$$

Proof: The following inequality [10] is used:

$$
0 \leq \int_{0}^{1} u^{2} d x-\left(\int_{0}^{1} u d x\right)^{2} \leq \frac{1}{2} \int_{0}^{1} x(1-x) u^{\prime 2} d x
$$

The inequality is reduced to an equality only if $u$ is constant. Assume that $u$ is given by (11), then $\int_{0}^{1} u d x=0$. From (27), equation $(26)$ is obtained. 
Further $\varsigma_{i} / D$ is called the upper bound number two (UB2). Note that $\frac{1}{2} x_{i}\left(1-x_{i}\right)$ for $0 \leq x_{i} \leq 1$ is bounded: $0 \leq \frac{1}{2} x_{i}\left(1-x_{i}\right) \leq 1 / 8$. Therefore, $0 \leq \varsigma_{i} \leq \nu_{i} / 8$.

\section{Normally distributed variables}

\section{Lower bound on $S_{i}^{\text {tot }}$}

Theorem 6. If $X_{i}$ is normally distributed with a mean $\mu_{i}$ and a finite variance $\sigma_{i}^{2}$, there exists the following lower bound between DGSM (7) and the Sobol' total sensitivity index:

$$
\frac{\sigma_{i}^{4}}{\left(\mu_{i}^{2}+\sigma_{i}^{2}\right) V} w_{i}^{2} \leq S_{i}^{\text {tot }}
$$

Proof: Using the equation (15) and Cauchy-Schwartz inequality applied on $\int_{R^{d}} x_{i} u_{i}(\mathbf{x}) d F(\mathbf{x})$ (with $F$ the joint cdf), Kucherenko and Song [16] give the proof of this inequality when $\mu_{i}=0$ (omitting to mention this condition). The general proof, obtained by Petit [25], is given below.

Consider a univariate function $g(X)$, with $X$ a normally distributed variable with mean $\mu$, finite variance $\sigma^{2}$ and cdf $F$. With adequate conditions on $g$, the following equality is obtained by integrating by parts:

$$
\begin{gathered}
\mathbb{E}\left[g^{\prime}(X)\right]=\int_{-\infty}^{\infty} g^{\prime}(x) d F(x)=\frac{1}{\sigma \sqrt{2 \pi}} \int_{-\infty}^{\infty} g^{\prime}(x) \exp \left[-\frac{(x-\mu)^{2}}{2 \sigma^{2}}\right] d x \\
=\frac{1}{\sigma \sqrt{2 \pi}}\left[g(x) \exp \left[-\frac{(x-\mu)^{2}}{2 \sigma^{2}}\right]\right]_{-\infty}^{+\infty}+\frac{1}{\sigma \sqrt{2 \pi}} \int_{-\infty}^{\infty} g(x) \frac{x-\mu}{\sigma^{2}} \exp \left[-\frac{(x-\mu)^{2}}{2 \sigma^{2}}\right] d x \\
=\frac{1}{\sigma^{2}} \int_{-\infty}^{\infty} x g(x) d F(x)-\mu \int_{-\infty}^{\infty} g(x) d F(x) .
\end{gathered}
$$

In this equation, replacing $g(x)$ by $u_{i}(\mathbf{x})$ with $x_{i}$ normally distributed, the $w_{i}$ DGSM writes

$$
w_{i}=\int_{R^{d}} \frac{\partial G(\mathbf{x})}{\partial x_{i}} d F(\mathbf{x})=\int_{R^{d}} \frac{\partial u_{i}(\mathbf{x})}{\partial x_{i}} d F(\mathbf{x})=\frac{1}{\sigma_{i}^{2}} \int_{R^{d}} x_{i} u_{i}(\mathbf{x}) d F(\mathbf{x}),
$$

because $\int_{R^{d}} u_{i}(\mathbf{x}) d F(\mathbf{x})=0$ (due to the ANOVA decomposition condition). Moreover, the Cauchy-Schwartz inequality applied on $\int_{R^{d}} x_{i} u_{i}(\mathbf{x}) d F(\mathbf{x})$ gives 


$$
\left[\int_{R^{d}} x_{i} u_{i}(\mathbf{x}) d F(\mathbf{x})\right]^{2} \leq \int_{R^{d}} x_{i}^{2} d F(\mathbf{x}) \int_{R^{d}}\left[u_{i}(\mathbf{x})\right]^{2} d F(\mathbf{x})
$$

Combining the two latter equations leads to the expression

$$
w_{i}^{2} \leq \frac{1}{\sigma_{i}^{4}}\left(\mu_{i}^{2}+\sigma_{i}^{2}\right) V S_{i}^{\text {tot }}
$$

which is equivalent to Eq. 28.

\section{Upper bounds on $S_{i}^{\text {tot }}$}

The following Theorem 7 is a generalization of Theorem 1.

Theorem 7. If $X_{i}$ has a finite variance $\sigma_{i}^{2}$ and $c \leq\left|\frac{\partial G}{\partial x_{i}}\right| \leq C$, then

$$
\frac{\sigma_{i}^{2} c^{2}}{V} \leq S_{i}^{\text {tot }} \leq \frac{\sigma_{i}^{2} C^{2}}{V}
$$

The constant factor $\sigma_{i}^{2}$ cannot be improved.

Theorem 8. If $X_{i}$ is normally distributed with a finite variance $\sigma_{i}^{2}$, there exists the following upper bound between DGSM (6) and the Sobol' total sensitivity index:

$$
S_{i}^{\text {tot }} \leq \frac{\sigma_{i}^{2}}{V} \nu_{i}
$$

The constant factor $\sigma_{i}^{2}$ cannot be reduced.

Proof: The proofs of these Theorems are presented in Sobol and Kucherenko [38].

\section{DGSM-based bounds for groups of variables}

Let $\mathbf{x}=\left(x_{1}, \ldots, x_{d}\right)$ be a point in the $d$-dimensional unit hypercube with Lebesgue measure $d \mathbf{x}=d x_{1} \cdots d x_{d}$. Consider an arbitrary subset of the variables $y=\left(x_{i_{1}}, \ldots, x_{i_{s}}\right)$, $1 \leq i_{1} \leq \ldots \leq i_{s} \leq d$, and the set of remaining complementary variables $z$, so that $\mathbf{x}=(y, z), d \mathbf{x}=d y d z$. Further all the integrals are written without integration limits, by assuming that each integration variable varies independently from 0 to 1 . 
Consider the following DGSM $\tau_{y}$ :

$$
\tau_{y}=\sum_{p=1}^{s} \int\left(\frac{\partial G(\mathbf{x})}{\partial x_{i_{p}}}\right)^{2} \frac{1-3 x_{i_{p}}+3 x_{i_{p}}^{2}}{6} d \mathbf{x} .
$$

Theorem 9. If $G(\mathbf{x})$ is linear with respect to $x_{i_{1}}, \ldots, x_{i_{s}}$, then $V_{y}^{\text {tot }}=\tau_{y}$, or in other words $S_{y}^{\text {tot }}=\frac{\tau_{y}}{V}$.

Theorem 10. The following general inequality holds: $V_{y}^{\text {tot }} \leq\left(24 / \pi^{2}\right) \tau_{y}$, or in other words $S_{y}^{\text {tot }} \leq \frac{24}{\pi^{2} V} \tau_{y}$.

Proof: The proofs of these Theorems are given in Sobol and Kucherenko [39]. The second theorem shows that small values of $\tau_{y}$ imply small values of $S_{y}^{\text {tot }}$ and this allows identification of a set of unessential factors $y$ (usually defined by a condition of the type $S_{y}^{\text {tot }}<\epsilon$, where $\epsilon$ is small).

\section{Importance criterion $\tau_{i}$}

Consider the one dimensional case when the subset $y$ consists of only one variable $y=\left(x_{i}\right)$, then measure $\tau_{y}=\tau_{i}$ has the form

$$
\tau_{i}=\int\left(\frac{\partial G(\mathbf{x})}{\partial x_{i}}\right)^{2} \frac{1-3 x_{i}+3 x_{i}^{2}}{6} d \mathbf{x} .
$$

It is easy to show that $\nu_{i} / 24 \leq \tau_{i} \leq \nu_{i} / 6$. From UB1 it follows that

$$
S_{i}^{\text {tot }} \leq \frac{24}{\pi^{2} V} \tau_{i}
$$

Thus small values of $\tau_{i}$ imply small values of $S_{i}^{\text {tot }}$, that are characteristic for non important variables $x_{i}$. At the same time, the following corollary is obtained from Theorem 9: if $G(\mathbf{x})$ depends linearly on $x_{i}$, then $S_{i}^{\text {tot }}=\tau_{i} / V$. Thus $\tau_{i}$ is closer to $V_{i}^{\text {tot }}$ than $\nu_{i}$.

Note that the constant factor $1 / \pi^{2}$ in 25 is the best possible. But in the general inequality for $\tau_{i}(33)$ the best possible constant factor is unknown.

There is a general link between importance measures $\tau_{i}, \varsigma_{i}$ and $\nu_{i}$ : 


$$
\tau_{i}=-\varsigma_{i}+\frac{1}{6} \nu_{i}
$$

then

$$
\varsigma_{i}=\frac{1}{6} \nu_{i}-\tau_{i}
$$

\section{Normally distributed random variables}

Consider independent normal random variables $X_{1}, \ldots, X_{d}$ with parameters $\left(\mu_{i}, \sigma_{i}\right)_{i=1 \ldots d}$.

Define $\tau_{i}$ as

$$
\tau_{i}=\frac{1}{2} \mathbb{E}\left[\left(\frac{\partial G(\mathbf{x})}{\partial x_{i}}\right)^{2}\left(x_{i}^{\prime}-x_{i}\right)^{2}\right] .
$$

The expectation over $x_{i}^{\prime}$ can be computed analytically. Then

$$
\tau_{i}=\frac{1}{2} \mathbb{E}\left[\left(\frac{\partial G(\mathbf{x})}{\partial x_{i}}\right)^{2} \frac{\left(x_{i}-\mu_{i}\right)^{2}+\sigma_{i}^{2}}{2}\right]
$$

Theorem 11. If $X_{1}, \ldots, X_{d}$ are independent normal random variables, then for an arbitrary subset $y$ of these variables, the following inequality is obtained:

$$
S_{y}^{\text {tot }} \leq \frac{2}{V} \tau_{y}
$$

Proof: The proof is given in Sobol and Kucherenko [39].

\section{DGSM-based upper bounds in the general case}

As previously, consider the function $G\left(X_{1}, \ldots, X_{d}\right)$, where $X_{1}, \ldots, X_{d}$ are independent random variables, defined in the Euclidian space $R^{d}$, with cdfs $F_{1}\left(x_{1}\right), \ldots, F_{d}\left(x_{d}\right)$. Assume further that each $X_{i}$ admits a probability density function (pdf), denoted by $f_{i}\left(x_{i}\right)$. In the following, all the integrals are written without integration limits.

The developments in this section are based on the classical $L^{2}$-Poincaré inequality:

$$
\int G(\mathbf{x})^{2} d F(\mathbf{x}) \leq C(F) \int\|\nabla G(\mathbf{x})\|^{2} d F(\mathbf{x})
$$


where $F$ is the joint cdf of $\left(X_{1}, \ldots, X_{d}\right)$. (34) is valid for all functions $G$ in $L^{2}(F)$ such that $\int G(\mathbf{x}) d F(\mathbf{x})=0$ and $\|\nabla f\| \in L^{2}(F)$. The constant $C(F)$ in Eq. (34) is called a Poincaré constant of $F$. In some cases, it exists and optimal Poincaré constant $C_{\mathrm{opt}}(F)$ which is the best possible constant. In measure theory, the Poincaré constants are expressed as a function of so-called Cheeger constants [1] which are used for SA in Lamboni et al [19] (see Roustant et al [28] for more details).

A connection between total indices and DGSM has been established by Lamboni et al [19] for variables with continuous distributions (called Boltzmann probability measures in their paper).

Theorem 12. Let $F_{i}$ and $f_{i}$ be respectively the cdf and the pdf of $X_{i}$, the following inequality is obtained:

$$
S_{i}^{\text {tot }} \leq \frac{C\left(F_{i}\right)}{V} \nu_{i}
$$

with $\nu_{i}$ the DGSM defined in Eq. (6) and

$$
C\left(F_{i}\right)=4\left[\sup _{x \in \mathbb{R}} \frac{\min \left(F_{i}(x), 1-F_{i}(x)\right)}{f_{i}(x)}\right]^{2} .
$$

Proof: This result comes from the direct application of the $L^{2}$-Poincaré inequality 34 on $u_{i}(\mathbf{x})$ (see Eq. (11))

In Lamboni et al [19] and Roustant et al [28], the particular case of log-concave probability distribution has been developed. It includes classical distributions as for instance the normal, exponential, Beta, Gamma and Gumbel distributions. In this case, the constant writes

$$
C\left(F_{i}\right)=\frac{1}{f_{i}\left(\tilde{m}_{i}\right)^{2}}
$$

with $\tilde{m}_{i}$ the median of the distribution $F_{i}$. This allows to obtain analytical expressions for $C\left(F_{i}\right)$ in several cases [19]. In the case of a log-concave truncated distribution on $[a, b]$, the constant writes [28]

$$
\left(F_{i}(b)-F_{i}(a)\right)^{2} / f_{i}\left(q_{i}\left(\frac{F_{i}(a)+F_{i}(b)}{2}\right)\right)^{2}
$$


with $q_{i}(\cdot)$ the quantile function of $X_{i}$. Table 1 gives some examples of Poincaré constants for several well-known and often used probability distributions in practice.

\begin{tabular}{lcc}
\hline Distribution & Poincaré constant & Optimal constant \\
\hline Uniform $\mathcal{U}[a b]$ & $(b-a)^{2} / \pi^{2}$ & yes \\
Normal $\mathcal{N}\left(\mu, \sigma^{2}\right)$ & $\sigma^{2}$ & yes \\
Exponential $\mathcal{E}(\lambda), \lambda>0$ & $\frac{4}{\lambda^{2}}$ & yes \\
Gumbel $\mathcal{G}(\mu, \beta)$, scale $\beta>0$ & $\left(\frac{2 \beta}{\log 2}\right)^{2}$ & no \\
Weibull $\mathcal{W}(k, \lambda)$, shape $k \geq 1$, scale $\lambda>0$ & {$\left[\frac{2 \lambda(\log 2)^{(1-k) / k}}{k}\right]^{2}$} & no \\
\hline
\end{tabular}

Table 1. Poincaré constants for a few probability distributions.

For studying second-order interactions, Roustant et al [28] have derived a similar to (35) inequality based on the squared crossed derivatives of the function. Assuming that second-order derivatives of $G$ are in $L^{2}(F)$, it uses the so-called crossed-DGSM

$$
\nu_{i j}=\int\left(\frac{\partial^{2} G(\mathbf{x})}{\partial x_{i} \partial x_{j}}\right)^{2} d F(\mathbf{x})
$$

introduced by Friedman and Popescu [7]. An inequality link is made with an extension of the total Sobol' sensitivity indices to general sets of variables (called superset importance or total interaction index) proposed by Liu and Owen [20]. In the case of a pair of variables $\left\{X_{i}, X_{j}\right\}$, the superset importance is defined as

$$
V_{i j}^{\text {super }}=\sum_{I \supseteq\{i, j\}} V_{I}
$$

The estimation methods of this total interaction index have also been studied by Fruth et al [8].

Theorem 13. For all pairs $\{i, j\}(1 \leq i<j \leq d)$,

$$
V_{i j} \leq V_{i j}^{\text {super }} \leq C\left(F_{i}\right) C\left(F_{j}\right) \nu_{i j}
$$

These inequalities with the corresponding Sobol' indices write 


$$
S_{i j} \leq S_{i j}^{\text {super }} \leq \frac{C\left(F_{i}\right) C\left(F_{j}\right)}{V} \nu_{i j}
$$

Roustant et al 28] have shown on several examples how to apply this result in order to detect pairs of inputs that do not interact together (see also Muehlenstaedt et al [22] and Fruth et al 8] which use Sobol' indices).

\section{Computational costs}

All DGSM can be computed using the same set of partial derivatives $\frac{\partial G(\mathbf{x})}{\partial x_{i}}, i=$ $1, \ldots, d$. Evaluation of $\frac{\partial G(\mathbf{x})}{\partial x_{i}}$ can be done analytically for explicitly given easilydifferentiable functions or numerically:

$$
\frac{\partial G\left(\mathbf{x}^{*}\right)}{\partial x_{i}}=\frac{\left[G\left(x_{1}^{*}, \ldots, x_{i-1}^{*}, x_{i}^{*}+\delta, x_{i+1}^{*}, \ldots, x_{n}^{*}\right)-G\left(\mathbf{x}^{*}\right)\right]}{\delta} .
$$

This is called a finite-difference scheme (see Variational Methods) with $\delta$ which is a small increment. There is a similarity with the elementary effect formula (2) of the Morris method which is however computed with large $\Delta$.

In the case of straightforward numerical estimations of all partial derivatives (43) and computation of integrals using MC or QMC methods, the number of required function evaluations for a set of all input variables is equal to $N(d+1)$, where $N$ is a number of sampled points. Computing LB1 also requires values of $G(0, \mathbf{z}), G(1, \mathbf{z})$, while computing LB2 requires only values of $G(1, \mathbf{z})$. In total, numerical computation of $\mathrm{LB}^{*}$ for all input variables would require $N_{G}^{\mathrm{LB}}=N(d+1)+2 N d=N(3 d+1)$ function evaluations. Computation of all upper bounds require $N_{G}^{\mathrm{UB}}=N(d+1)$ function evaluations. This is the same number that the number of function evaluations required for computation of $S_{i}^{\text {tot }}$ which is $N_{G}^{S}=N(d+1)$ [31].

However, the number of sampled points $N$ needed to achieve numerical convergence can be different for DGSM and $S_{i}^{\text {tot }}$. It is generally lower for the case of 
DGSM. Moreover, the numerical efficiency of the DGSM method can be significantly increased by using algorithmic differentiation in the adjoint (reverse) mode [9] (see also Variational Methods). This approach allows estimating all derivatives at a cost independent of $d$, at most 4-6 times of that for evaluating the original function $G(\mathbf{x})$ 13 .

\section{Test cases}

In this section, three test cases are considered, in order to illustrate application of DGSM and their links with $S_{i}^{\text {tot }}$.

Example 1. Consider a linear with respect to $x_{i}$ function:

$$
G(x)=a(\mathbf{z}) x_{i}+b(\mathbf{z})
$$

For this function $S_{i}=S_{i}^{\text {tot }}, V_{i}^{\text {tot }}=\frac{1}{12} \int_{H^{d-1}} a^{2}(\mathbf{z}) d \mathbf{z}, \nu_{i}=\int_{H^{d-1}} a^{2}(\mathbf{z}) d \mathbf{z}$, LB1 $=$ $\frac{\left(\int_{H^{d}}\left(a^{2}(\mathbf{z})-2 a^{2}(\mathbf{z}) x_{i}\right) d \mathbf{z} d x_{i}\right)^{2}}{4 V \int_{H^{d-1}} a^{2}(\mathbf{z}) d \mathbf{z}}=0$ and $\gamma(m)=\frac{(2 m+1) m^{2}\left(\int_{H^{d-1}} a(\mathbf{z}) d \mathbf{z}\right)^{2}}{4(m+2)^{2}(m+1)^{2} V}$. A maximum value of $\gamma(m)$ is attained at $m^{*}=3.745$, while $\gamma^{*}\left(m^{*}\right)=\frac{0.0401}{V}\left(\int a(\mathbf{z}) d \mathbf{z}\right)^{2}$. The lower and upper bounds are $\mathrm{LB}^{*} \approx 0.48 S_{i}^{\mathrm{tot}}, \mathrm{UB} 1 \approx 1.22 S_{i}^{\mathrm{tot}} . \mathrm{UB} 2=\frac{1}{12 \mathrm{~V}} \int_{0}^{1} a(\mathbf{z})^{2} d \mathbf{z}=$ $S_{i}^{\text {tot }}$

For this test function UB2 $<$ UB1.

Example 2. Consider the so-called g-function which is often used in global SA for illustration purposes:

$$
G(x)=\prod_{i=1}^{d} v_{i}
$$

where $v_{i}=\frac{\left|4 x_{i}-2\right|+a_{i}}{1+a_{i}}, a_{i}(i=1, \ldots, d)$ are constants. It is easy to see that for this function $g_{i}\left(x_{i}\right)=\left(v_{i}-1\right), u_{i}(x)=\left(v_{i}-1\right) \prod_{j=1, j \neq i}^{d} v_{j}$ and as a result LB1 $=0$. The total variance is $V=-1+\prod_{j=1}^{d}\left(1+\frac{1 / 3}{\left(1+a_{j}\right)^{2}}\right)$. The analytical values of $S_{i}, S_{i}^{\text {tot }}$ and LB2 are given in Table 2 , 
Table 2. The analytical expressions for $S_{i}, S_{i}^{\text {tot }}$ and LB2 for g-function.

\begin{tabular}{|c|c|c|}
\hline$S_{i}$ & $S_{i}^{\text {tot }}$ & $\gamma(m)$ \\
\hline$\frac{1 / 3}{\left(1+a_{i}\right)^{2} V}$ & $\frac{\frac{1 / 3}{\left(1+a_{i}\right)^{2}} \prod_{j=1, j \neq i}^{d}\left(1+\frac{1 / 3}{\left(1+a_{j}\right)^{2}}\right)}{V}$ & $\frac{(2 m+1)\left[1-\frac{4\left(1-(1 / 2)^{m+1}\right)}{m+2}\right]^{2}}{\left(1+a_{i}\right)^{2}(m+1)^{2} V}$ \\
\hline
\end{tabular}

By solving equation $\frac{d \gamma(m)}{d m}=0, m^{*}=9.64$ and $\gamma\left(m^{*}\right)=\frac{0.0772}{\left(1+a_{i}\right)^{2} V}$. It is interesting to note that $m^{*}$ does not depend on $a_{i}, i=1,2, \ldots, d$ and $d$. In the extreme cases: if $a_{i} \rightarrow \infty$ for all $i, \frac{\gamma\left(m^{*}\right)}{S_{i}^{\text {tot }}} \rightarrow 0.257, \frac{S_{i}}{S_{i}^{\text {tot }}} \rightarrow 1$, while if $a_{i} \rightarrow 0$ for all $i$, $\frac{\gamma\left(m^{*}\right)}{S_{i}^{\text {tot }}} \rightarrow \frac{0.257}{(4 / 3)^{d-1}}, \frac{S_{i}}{S_{i}^{\text {tot }}} \rightarrow \frac{1}{(4 / 3)^{d-1}}$. The analytical expression for $S_{i}^{\text {tot }}$, UB1 and UB2 are given in Table 3 .

Table 3. The analytical expressions for $S_{i}^{\text {tot }}$, UB1 and UB2 for g-function.

\begin{tabular}{|c|c|c|}
\hline$S_{i}^{\text {tot }}$ & $\mathrm{UB} 1$ & $\mathrm{UB} 2$ \\
\hline$\frac{\frac{1 / 3}{\left(1+a_{i}\right)^{2}} \prod_{j=1, j \neq i}^{d}\left(1+\frac{1 / 3}{\left(1+a_{j}\right)^{2}}\right)}{V}$ & $\frac{16 \prod_{j=1, j \neq i}^{d}\left(1+\frac{1 / 3}{\left(1+a_{j}\right)^{2}}\right)}{\left(1+a_{i}\right)^{2} \pi^{2} V}$ & $\frac{4 \prod_{j=1, j \neq i}^{d}\left(1+\frac{1 / 3}{\left(1+a_{j}\right)^{2}}\right)}{3\left(1+a_{i}\right)^{2} V}$ \\
\hline
\end{tabular}

For this test function $\frac{S_{i}^{\text {tot }}}{\mathrm{UB} 1}=\frac{\pi^{2}}{48}, \frac{S_{i}^{\mathrm{tot}}}{\mathrm{UB} 2}=\frac{1}{4}$, hence $\frac{\mathrm{UB} 2}{\mathrm{UB} 1}=\frac{\pi^{2}}{12}<1$.

Values of $S_{i}, S_{i}^{\text {tot }}, \mathrm{UB} 1, \mathrm{UB} 2$ and LB2 for the case of $\boldsymbol{a}=[0,1,4.5,9,99,99,99,99]$, $d=8$ are given in Table 4 and shown in Fig. 1. One can see that the knowledge of LB2 and UB1 allows to rank correctly all the variables in the order of their importance.

Table 4. Values of LB* $S_{i}, S_{i}^{\text {tot }}$, UB1 and UB1. Example $2, \boldsymbol{a}=[0,1,4.5,9,99,99,99,99], d=8$.

\begin{tabular}{|c|c|c|c|c|c|}
\hline & $x_{1}$ & $x_{2}$ & $x_{3}$ & $x_{4}$ & $x_{5} \ldots x_{8}$ \\
\hline $\mathrm{LB}^{*}$ & 0.166 & 0.0416 & 0.00549 & 0.00166 & 0.000017 \\
\hline$S_{i}$ & 0.716 & 0.179 & 0.0237 & 0.00720 & 0.0000716 \\
\hline$S_{i}^{\text {tot }}$ & 0.788 & 0.242 & 0.0343 & 0.0105 & 0.000105 \\
\hline $\mathrm{UB} 1$ & 3.828 & 1.178 & 0.167 & 0.0509 & 0.00051 \\
\hline $\mathrm{UB} 2$ & 3.149 & 0.969 & 0.137 & 0.0418 & 0.00042 \\
\hline
\end{tabular}

Example 3. Consider the reduced Morris' test function with four inputs [3]: 


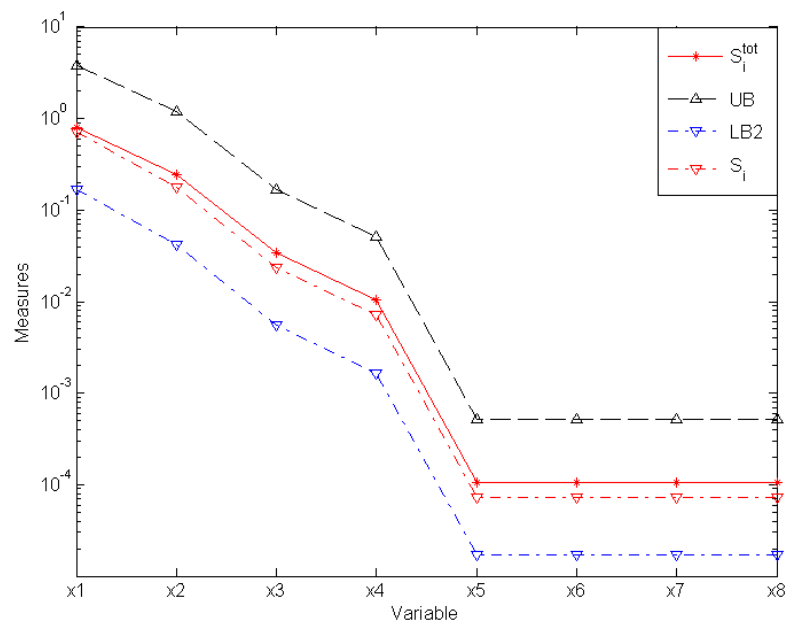

Fig. 1. Values of $S_{i}, S_{i}^{\text {tot }}, \mathrm{LB} 2$ and UB1 for all input variables. Example 2 with $\mathbf{a}=$ $[0,1,4.5,9,99,99,99,99], d=8$.

$$
f(\boldsymbol{x})=\sum_{i=1}^{4} b_{i} x_{i}+\sum_{i \leq j}^{4} b_{i j} x_{i} x_{j}+\sum_{i \leq j \leq k}^{4} b_{i j k} x_{i} x_{j} x_{k}
$$

$$
\text { with } b_{i}=\left[\begin{array}{c}
0.05 \\
0.59 \\
10 \\
0.21
\end{array}\right] \quad, \quad b_{i j}=\left[\begin{array}{cccc}
0 & 80 & 60 & 40 \\
0 & 30 & 0.73 & 0.18 \\
0 & 0 & 0.64 & 0.93 \\
0 & 0 & 0 & 0.06
\end{array}\right], \quad b_{i j 4}=\left[\begin{array}{cccc}
0 & 10 & 0.98 & 0.19 \\
0 & 0 & 0.49 & 50 \\
0 & 0 & 0 & 1 \\
0 & 0 & 0 & 0
\end{array}\right] \text {. }
$$

The indices $b_{i j k} \forall k \neq 4$ are null.

The four input variables $x_{i}(i=1, \ldots, 4)$ follow uniform distribution on $[0,1]$. Sobol' indices are computed via the Monte-carlo scheme of Saltelli [29] (using two initial matrices of size $10^{5}$ ), while DGSM are computed with Monte-Carlo sampling of size $n$ (using derivatives computing by finite differences (43) with $\delta=10^{-5}$ ), with $n$ ranging from 20 to 500 , Figure 2 shows that DGSM bounds $\mathrm{UB} 1_{i}$ are greater than the total Sobol' indices $S_{T_{i}}$ (for $i=1,2,3,4$ ) as expected, except for $n<30$ which is a too small sample size. For small $S_{T_{i}}, \mathrm{UB} 1_{i}$ is close to the $S_{T_{i}}$ value. It confirms that DGSM bounds are first useful for screening exercises. Other numerical tests involving non-uniform and non-normal distributions for the inputs can be found in Lamboni et al [19] and Fruth et al [8]. 


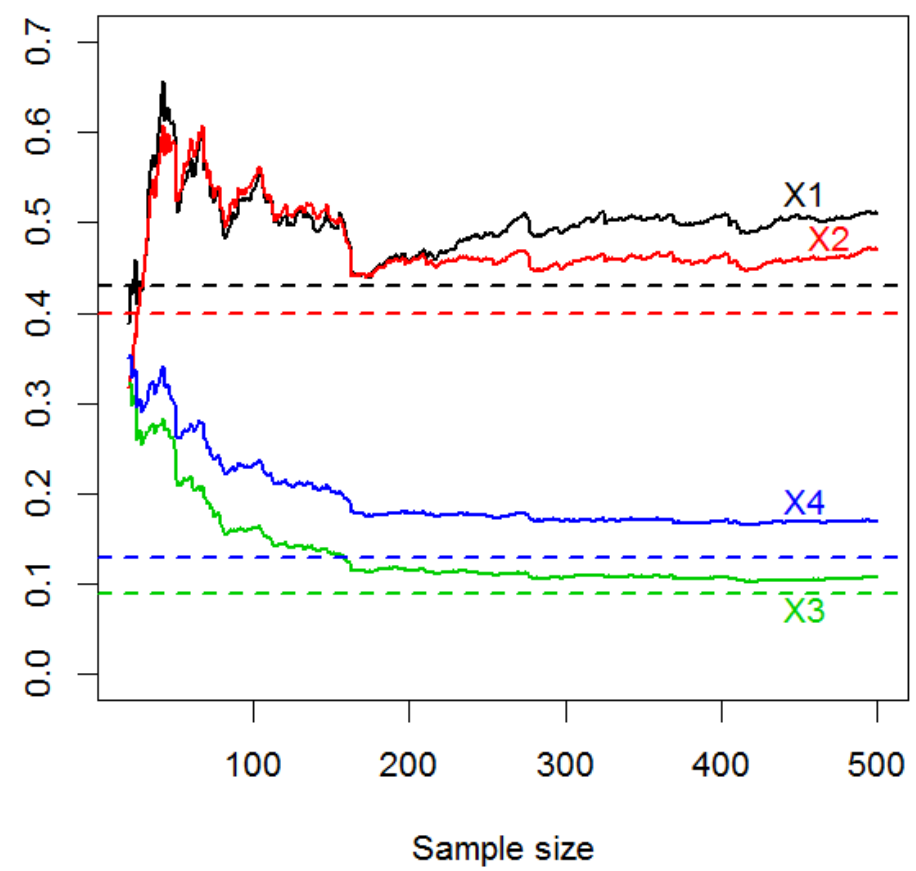

Fig. 2. For the 4 input variables of the reduced Morris' test function: Convergence of the DGSM bound estimates (solid lines) in function of the sample size and comparison to theoretical values of total Sobol' indices $S_{T_{i}}$ (dashed lines).

\section{Conclusions}

This paper has shown that using lower and upper bounds based on DGSM is possible in most cases to get a good practical estimation of the values of $S_{i}^{\text {tot }}$ at a fraction of the CPU cost for estimating $S_{i}^{\text {tot }}$. Upper and lower bounds can be estimated using $\mathrm{MC} / \mathrm{QMC}$ integration methods using the same set of partial derivative values. Most of the applications show that DGSM can be used for fixing unimportant variables and subsequent model reduction because small values of DGSM imply small values of $S_{i}^{\text {tot. }}$. In a general case variable ranking can be different for DGSM and variance based methods but for linear function and product function, DGSM can give the same variable ranking as $S_{i}^{\text {tot }}$. 
Engineering applications of DGSM can be found for instance in Kiparissides et al [15] and Rodriguez-Fernandez et al [27] for biological systems modeling, Patelli et al [24] for structural mechanics, Iooss et al [12] for an aquatic prey-predator model, Petit [25] for a river flood model and Touzany and Busby [41] for an hydrogeological simulator of the oil industry. One of the main prospect in practical situations is to use algorithmic differentiation in the reverse (adjoint) mode on the numerical model, allowing to estimate efficiency all partial derivatives of this model (see Variational

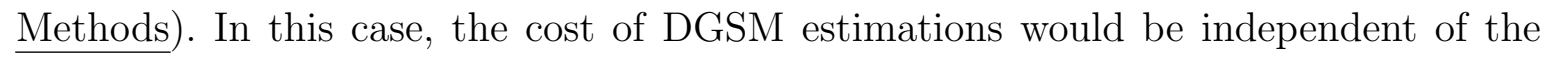
number of input variables. Obtaining global sensitivity information in a reasonable cpu time cost is therefore possible even for large-dimensional model (several tens and spatially distributed inputs in the recent and pioneering attempt of Petit [25]). When the adjoint model is not available, the DGSM estimation remains a problem in high dimension and novel ideas have to be explored [23] [24]. Coupling DGSM with nonparametric regression techniques or metamodel-based technique (see Metamodel-based sensitivity analysis: Polynomial chaos expansions and Gaussian processes) is another research prospect as first shown by Sudret and Mai [40] and De Lozzo and Marrel [5].

The authors would like to thank Prof. I. Sobol', Dr. S. Song, S. Petit, Dr. M. Lamboni, Dr. O. Roustant and Prof. F. Gamboa for their contributions to this work. One of the authors (SK) gratefully acknowledges the financial support by the EPSRC grant EP/H03126X/1.

\section{References}

1. Bobkov SG (1999) Isoperimetric and analytic inequalities for log-concave probability measures. The Annals of Probability 27(4):1903-1921

2. Campolongo F, Braddock R (1999) The use of graph theory in the sensitivity analysis of model output: a second order screening method. Reliability Engineering and System Safety 64:1-12 
3. Campolongo F, Cariboni J, Saltelli A (2007) An effective screening design for sensitivity analysis of large models. Environmental Modelling and Software 22:1509-1518

4. Cropp R, Braddock R (2002) The new Morris method: an efficient second-order screening method. Reliability Engineering and System Safety 78:77-83

5. De Lozzo M, Marrel A (2015) Estimation of the derivative-based global sensitivity measures using a Gaussian process metamodel. Submitted

6. Fédou JM, Rendas MJ (2015) Extending Morris method: identification of the interaction graph using cycle-equitable designs. Journal of Statistical Computation and Simulation 85:1398-1419

7. Friedman J, Popescu B (2008) Predictive Learning via Rule Ensembles. The Annals of Applied Statistics 2(3):916-954

8. Fruth J, Roustant O, Kuhnt S (2014) Total interaction index: A variance-based sensitivity index for second-order interaction screening. Journal of Statistical Planning and Inference 147:212-223

9. Griewank A, Walther A (2008) Evaluating derivatives: Principles and techniques of automatic differentiation. SIAM Philadelphia

10. Hardy G, Littlewood J, Polya G (1973) Inequalities. Cambridge University Press, Second edition

11. Homma T, Saltelli A (1996) Importance measures in global sensitivity analysis of non linear models. Reliability Engineering and System Safety 52:1-17

12. Iooss B, Popelin AL, Blatman G, Ciric C, Gamboa F, Lacaze S, Lamboni M (2012) Some new insights in derivative-based global sensitivity measures. In: Proceedings of the PSAM11 ESREL 2012 Conference, Helsinki, Finland, pp 1094-1104

13. Jansen K, Leovey H, Nube A, Griewank A, Mueller-Preussker M (2014) A first look of quasi-Monte Carlo for lattice field theory problems. Computer Physics Communication 185:948-959

14. Jansen M (1999) Analysis of variance designs for model output. Computer Physics Communication $117: 25-43$

15. Kiparissides A, Kucherenko S, Mantalaris A, Pistikopoulos E (2009) Global sensitivity analysis challenges in biological systems modeling. Journal of Industrial and Engineering Chemistry Research 48:1135-1148

16. Kucherenko S, Song S (2015) Derivative-based global sensitivity measures and their link with Sobol' sensitivity indices. In: Cools R, Nuyens D (eds) Proceedings of the Eleventh International Conference on Monte Carlo and Quasi-Monte Carlo Methods in Scientific Computing (MCQMC 2014), Springer-Verlag,, Leuven, Belgium 
17. Kucherenko S, Rodriguez-Fernandez M, Pantelides C, Shah N (2009) Monte carlo evaluation of derivative-based global sensitivity measures. Reliability Engineering and System Safety 94:11351148

18. Lamboni M (2013) New way of estimating total sensitivity indices. In: Proceedings of the 7 th International Conference on Sensitivity Analysis of Model Output (SAMO 2013), Nice, France

19. Lamboni M, Iooss B, Popelin AL, Gamboa F (2013) Derivative-based global sensitivity measures: general links with sobol' indices and numerical tests. Mathematics and Computers in Simulation $87: 45-54$

20. Liu R, Owen A (2006) Estimating mean dimensionality of analysis of variance decompositions. Journal of the American Statistical Association 101(474):712-721

21. Morris M (1991) Factorial sampling plans for preliminary computational experiments. Technometrics 33:161-174

22. Muehlenstaedt T, Roustant O, Carraro L, Kuhnt S (2012) Data-driven Kriging models based on FANOVA-decomposition. Statistics \& Computing 22:723-738

23. Patelli E, Pradlwarter H (2010) Monte Carlo gradient estimation in high dimensions. International Journal for Numerical Methods in Engineering 81:172-188

24. Patelli E, Pradlwarter HJ, Schuëller GI (2010) Global sensitivity of structural variability by random sampling. Computer Physics Communications 181:2072-2081

25. Petit S (2015) Analyse de sensibilité globale du module MASCARET par l'utilisation de la différentiation automatique. Rapport de stage de fin d'études de Supélec, EDF R\&D, Chatou, France

26. Pujol G (2009) Simplex-based screening designs for estimating metamodels. Reliability Engineering and System Safety 94:1156-1160

27. Rodriguez-Fernandez M, Banga J, Doyle F (2012) Novel global sensitivity analysis methodology accounting for the crucial role of the distribution of input parameters: application to systems biology models. International Journal of Robust Nonlinear Control 22:1082-1102

28. Roustant O, Fruth J, Iooss B, Kuhnt S (2014) Crossed-derivative-based sensitivity measures for interaction screening. Mathematics and Computers in Simulation 105:105-118

29. Saltelli A (2002) Making best use of model evaluations to compute sensitivity indices. Computer Physics Communication 145:280-297 
30. Saltelli A, Ratto M, Andres T, Campolongo F, Cariboni J, Gatelli D, Salsana M, Tarantola S (2008) Global sensitivity analysis - The primer. Wiley

31. Saltelli A, Annoni P, Azzini I, Campolongo F, Ratto M, Tarantola S (2010) Variance based sensitivity analysis of model output. Design and estimator for the total sensitivity index. Computer Physics Communication 181:259-270

32. Santiago J, Corre B, Claeys-Bruno M, Sergent M (2012) Improved sensitivity through Morris extension. Chemometrics and Intelligent Laboratory Systems 113:52-57

33. Sobol I (1990) Sensitivity estimates for non linear mathematical models (in Russian). Matematicheskoe Modelirovanie 2:112-118

34. Sobol I (1993) Sensitivity estimates for non linear mathematical models. Mathematical Modelling and Computational Experiments 1:407-414

35. Sobol I (2001) Global sensitivity indices for non linear mathematical models and their Monte Carlo estimates. Mathematics and Computers in Simulation 55:271-280

36. Sobol I, Gershman A (1995) On an alternative global sensitivity estimators. In: Proceedings of SAMO 1995, Belgirate, pp 40-42

37. Sobol I, Kucherenko S (2005) Global sensitivity indices for non linear mathematical models. Review. Wilmott Magazine 1:56-61

38. Sobol I, Kucherenko S (2009) Derivative based global sensitivity measures and their links with global sensitivity indices. Mathematics and Computers in Simulation 79:3009-3017

39. Sobol I, Kucherenko S (2010) A new derivative based importance criterion for groups of variables and its link with the global sensitivity indices. Computer Physics Communications 181:12121217

40. Sudret B, Mai CV (2015) Computing derivative-based global sensitivity measures using polynomial chaos expansions. Reliability Engineering and System Safety 134:241-250

41. Touzany S, Busby D (2014) Screening method using the derivative-based global sensitivity indices with application to reservoir simulator. Oil \& Gas Science and Technology Rev IFP Energies nouvelles 69:619-632 